\title{
Homes of the Future
}

\author{
Bence Kovács ${ }^{1 *}$, János Tóth ${ }^{1}$ \\ ${ }^{1}$ University of Debrecen, Mechatronics Department, 4028 Debrecen Ótemető street 2-4., Hungary
}

\begin{abstract}
The industry is being revolutionised again. It is the fourth time, that is why this revolution is often called Fourth Industrial Revolution. One of the definitions of this process says that the aim is to digitalize the machines and resources and those should communicate with each other in the cyber-physical space via sensor networks and mobile communication. This automation process starts to appear in the households. People have the need to own fully automated houses and intelligent homes. In this article you can read about how a house can be intelligent.
\end{abstract}

\section{What does intelligent mean?}

If we use this phrase to humans, it is easy to tell what an "intelligent person" means. However, it is not that easy to define when we talk about houses and homes. Since it is the concept of the future, we cannot find exact definition of it. As I have mentioned in the Abstract, there are several definitions of the fourth industrial revolution and I had to choose my ideal one [1]. The case is the same with "intelligent houses". During my research I have found thoughts about being "intelligent" based on energy efficiency or the usage of renewable energy resources. Others say that a house can be called intelligent if it has an internal informatic network. Some say that an automated house is "Smart", but only when it is controlled by a central control unit, not separated, individual controls [2-4].

I do not agree with all of the statements, that is why I have decided to make my own criteria of "intelligent" and will build a model that meets the requirements and simulates real-life like conditions. I do not think that being "Smart" depends on energetic issues or renewable energies, but internal informatic network, automatic processes controlled by a central control unit and IoT. The automation should:

- make the owners life easier and more comfortable,

- provide security,

- have positive effects on (energy) consumption,

- $\quad$ and provide advanced fire alarm system.

\section{Selection of the central control unit}

I had to choose a control unit that could fulfil all four requirements of automation on its own. A Programmable Logic Controller (PLC) would have been a good choice, because it is usually manufactured to create automations. It usually has 24 VDC inputs and outputs, so it can directly control actuators and process sensor signals.
However, it requires specific knowledge to create complex controls and it is hard to fulfil IoT requirements. With the usage of a processor it is much easier to connect the whole house to the internet, but those chips are not designed for controlling power-hungry devices. And those have serial control.

My choice was a product of National Instruments. The control unit is a myRIO 1900. It has an integrated processor and a Field-Programmable Gate Array (FPGA) module. Thanks to the processor it is easy to connect the device to the internet and have real-time supervision, and to the FPGA I can achieve massive parallel control. It is a stand-alone application. The chosen controller can be seen on Fig. 1.

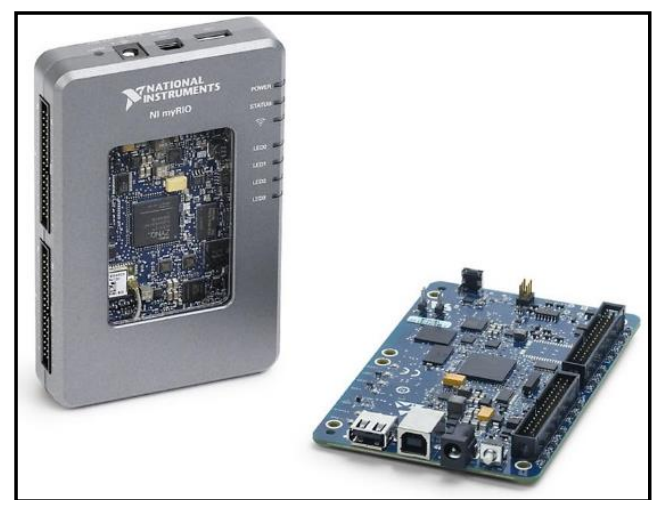

Fig. 1 - myRIO 1900 control unit

With this choice I am able to use the LabVIEW development platform provided by National Instruments. I can create my own programming code, the block diagrams and the user interface at the same time. Also, programming in g-code makes it more effective.

\section{Automatic controls}

My aim was to create:

- $\quad$ key-free access to the house,

\footnotetext{
* Bence Kovács: kovacs.bence0730@gmail.com
} 
- controlled lighting whit the option of moodlighting,

- motorized controls (Direct Current (DC) and Stepper),

- heating and cooling control,

- controlled sprinklers,

- $\quad$ security system with door/window opening and motion sensors,

- and an advance fire alarm system.

The most important aspect of an intelligent house for me is that the house should be aware of the presents of the owner and should create user-specific processes based on their needs.

I have achieved it by using Radio Frequency Identification (RFID) access control system. Each owner has an own card. The house senses their entry to the house and by using Universal Asynchronous ReceiverTransmitter (UART) communication protocol, it can recognise the individuals and perform pre-programmed user specific controls such as setting a specific room temperature or switching on mood-lightings. I had to use a hierarchical system among the users. If more persons are present and they have different needs, the control unit has to decide what to do. This decision making is based on my pre-programmed hierarchy. I have used a While loop with $10 \mathrm{~ms}$ timing for this part of the program. Each RFID card contains a 10 digit identification character. The program waits till all 10 of the characters arrive and then examines the code. I have used American Standard Code for Information Interchange (ASCII) character coding with 9600 baud rate, without parity bit. One stop bit registers the end of the communication. A portion of the RFID program can be seen on Fig. 2.

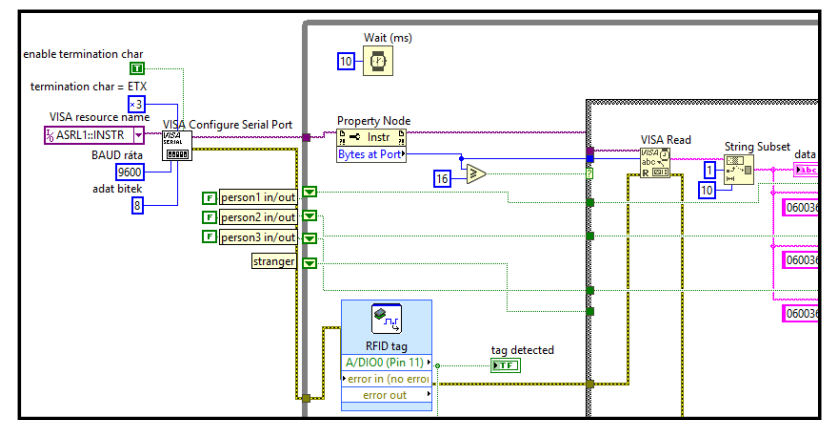

Fig. 2 - RFID program portion

Controlling lightings, motors, actuators and sensors was not that easy. Unfortunately, I had to make extra control circuits with amplifiers, because the myRIO can only provide $3.3 \mathrm{VDC}$ and $4 \mathrm{~mA}$. I used transistors, logiclevel metal-oxide-semiconductor field effect transistors (MOSFET) and relays for amplifying. I soldered everything together on universal printed circuit boards. An example of lighting control circuit with motion control can be seen on Fig. 3 .

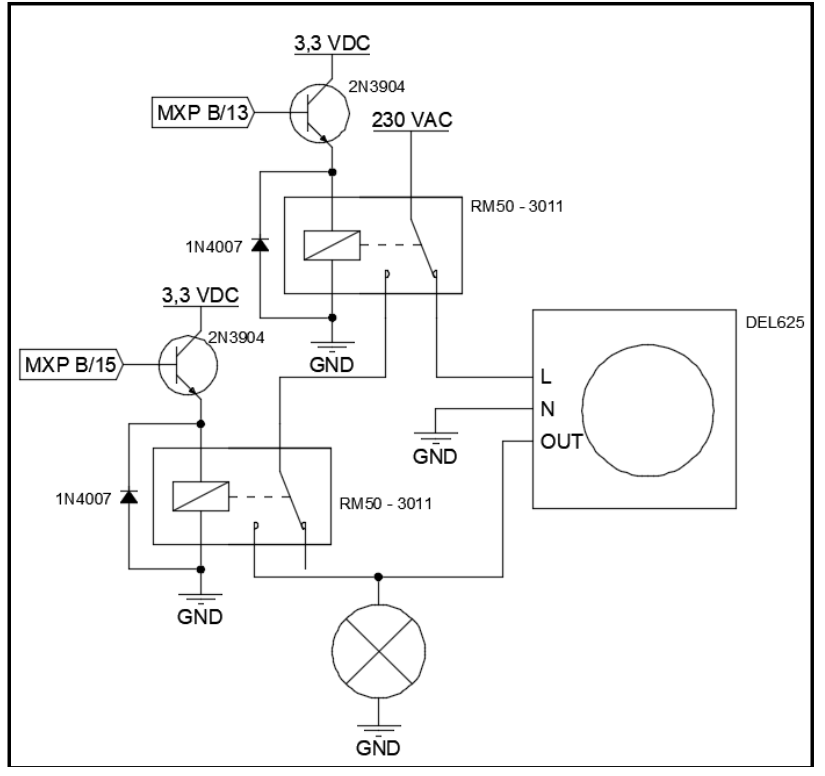

Fig. 3 - Additional control circuti for lighting

I have installed a wide variety of sensors to automate lighting. I have used ambient light sensors to detect the need of the lights to switch on. I have also used motion sensors and timers so that the house owners do not need to use switches. However they are able to overwrite any decision made by the controller. You can find several types of lights on the model, e.g. outdoor lights, indoor lights, standard bulbs, 12 VDC spot lights, light emitting diodes (LED), LED strips, etc. When I used MOSFET-s to control the brightness and the colour of my red-greenblue LED-s, it was important to use heatsinks as well. During the usage of lots of LED-s, amperes can go through the MOSFET-s and those produce significant heat. When applying heatsinks, I have to deal with electrical isolation between the Gate of the MOSFET (which is connected to the back area of the MOSFET) and the heatsink. Otherwise it would be dangerous to use.

The lights can be controlled manually (by switches) and via the Front Panel. A portion of the control program can be seen on Fig. 4.

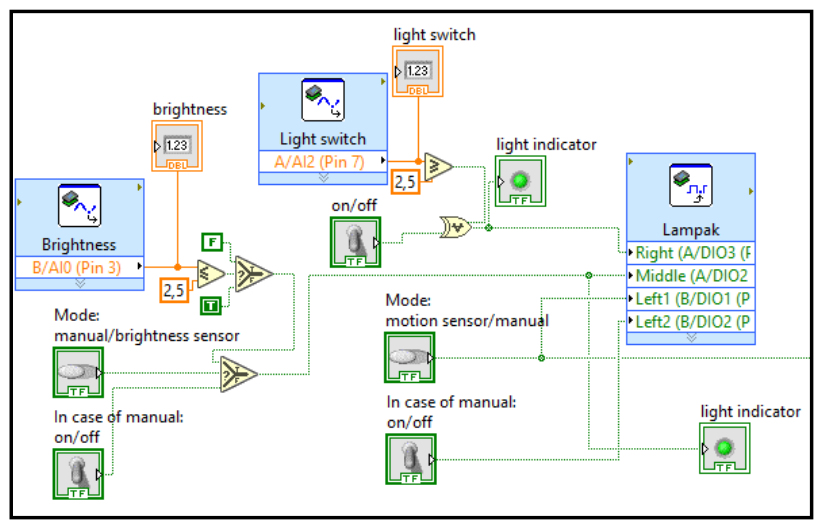

Fig. 4 - Portion of the lighting control program

There can be found an automatic sprinkler system in my intelligent home to make the user's life easier. It is time-controlled but the control unit detects the moisture of the soil and if it is wet, sprinkling will not happen. 
I have used two kind of motors to achieve controlled motions. For places where positioning is important, I have used stepper motors, otherwise I have used simple DC motors. The two kind requires different control methods, which I was able to program. The DC motor represents an automatic garage door. Two button is used on the user interface to "open" and "close" it. There is a light sensor for protection. If it signs during the closure of the door, the process is terminated.

The control of the stepper motor is more complicated. The owner of the house have the opportunity to vary the direction and the speed of the motor.

\section{Security functions}

For security purposes I have installed door and window opening sensors. If there is a robbery, a loud and unique alarm sound is activated. I have created the sound by programming Pulse Width Modulations (PWM). The frequency of the PWM is responsible for the tone, while the duty cycle is responsible for the uniqueness of the sound.

I have created other sound profiles for the RFID system. The access control system gives different sounds when it recognises and accepts a card and different ones when refuses them. This system also logs every movement. It logs when someone enters or leaves the home and saves it to a text file. This text file can be processed by any spreadsheet program. You can apply filtrations to get valuable information from the data with e.g. Excel. Fig. 5 shows and example.

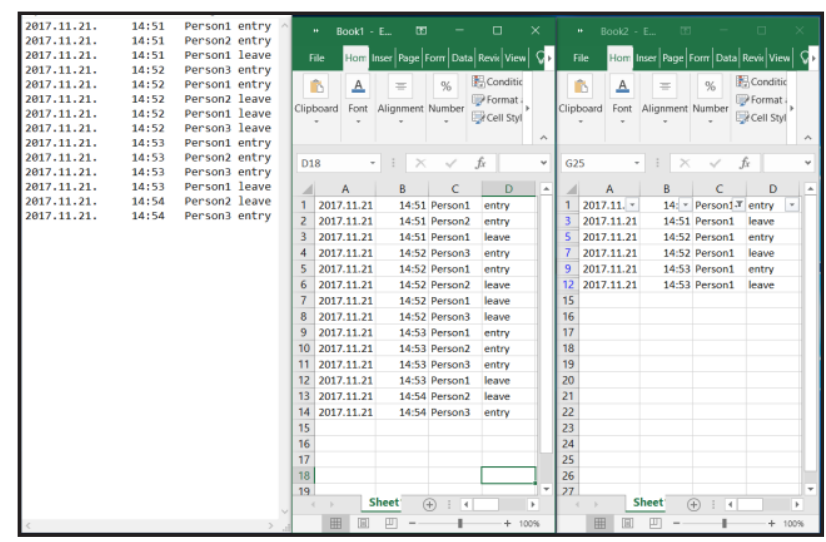

Fig. 5 - Movement logging and processing of the data

You can find an "intelligent" fire alarm system in my Smart home model. I have programmed the following functions to make it "intelligent":

- It has drift compensation. That means that by using counters, shift registers and logic values in my program, it can compensate the dust pollution of the sensor. However, after a while cleaning and maintenance of the sensors are compulsory.

- It will not provide false alarms. The signal of the sensors must be continuous for 30 seconds otherwise it is not accepted as fire and the system will not alarm.
- On the other hand, the system uses group decisions. If two or more neighbour sensors signs at the same time, there is no delay in the system, it is immediately activated. It is not the case when two, but not neighbour sensors signs.

- The users have the opportunity to check the fire as soon as possible, because they are notified via e-mail, and they receive a pop-up window on the front panel of the program, when there is a fire alarm.

A portion of the fire alarm program can be seen on Fig. 6.

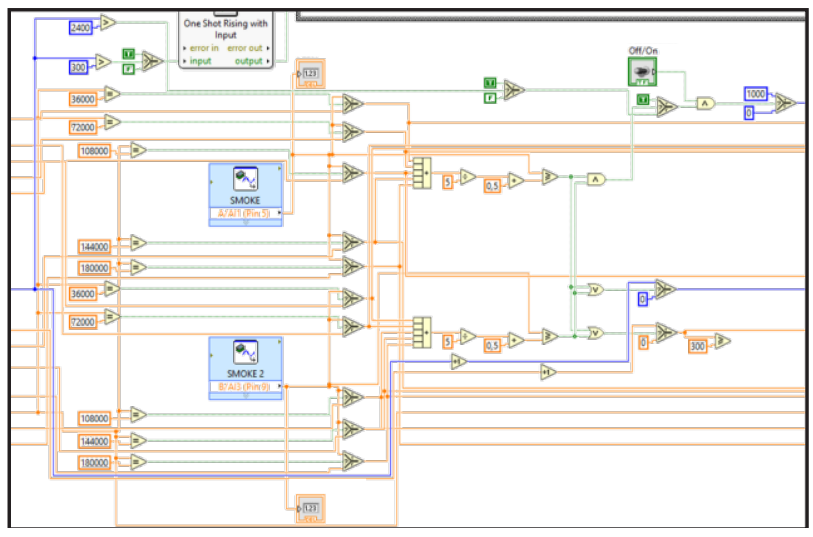

Fig. 6 - Protion of the intelligent fire alarm program

\section{Remote control}

I have connected my system to the internet. The myRIO has a built in Wireless Fidelity chip, so it can connect to a router wirelessly.

I have created a front panel in LabVIEW which can be seen on Fig. 7. The colour coding (e.g. yellow for lightings, blue for sprinkling, etc.) and the labels help the users to know what they are controlling. Also, indicators shows what is happening currently in the house.

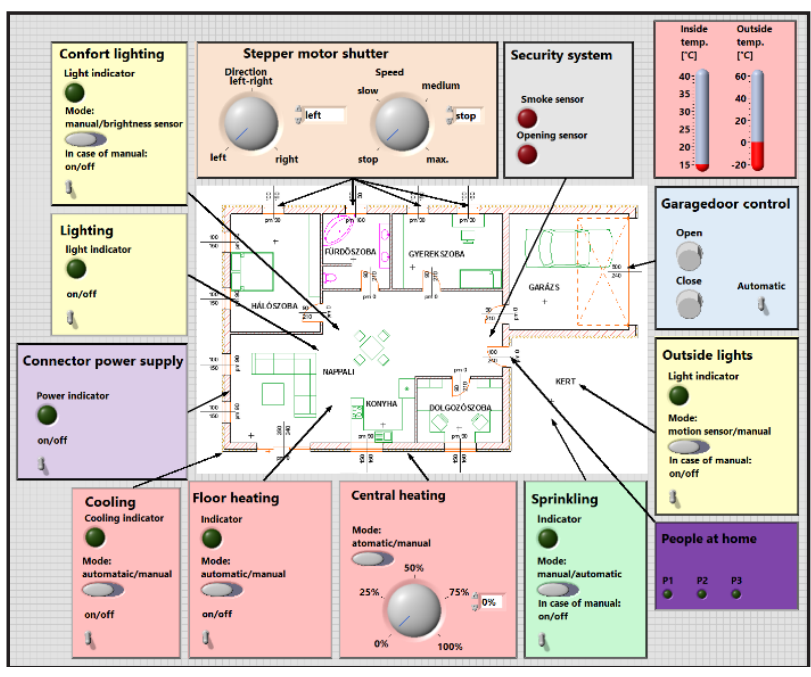

Fig. 7 - User interface / control panel

I have converted this front panel into a hypertext markup language (HTML) page and created a web server on the myRIO. 
With this technique, I am able to connect to the controller through the Internet. The only thing needed is a web browser.

\section{Built home model}

Last but not least I have built my home demonstration. I have used a big Oriented Standard Board as a base of my model. I wanted to have real-life like conditions, so I have used $230 \mathrm{VAC}$ and real sensors or actuators where I was able to. Every part of my automated processes can be found on the front face of the board, while the electronic components and the wiring is located on the back. On Fig. 8. you can find on these specific locations:
(1) lightings,
(2) controlled consumer electronics,
(3) security system,
(4) motorized controls,
(5) controlled sprinklers,
(6) key-free access control system,
(7) heating and cooling control.

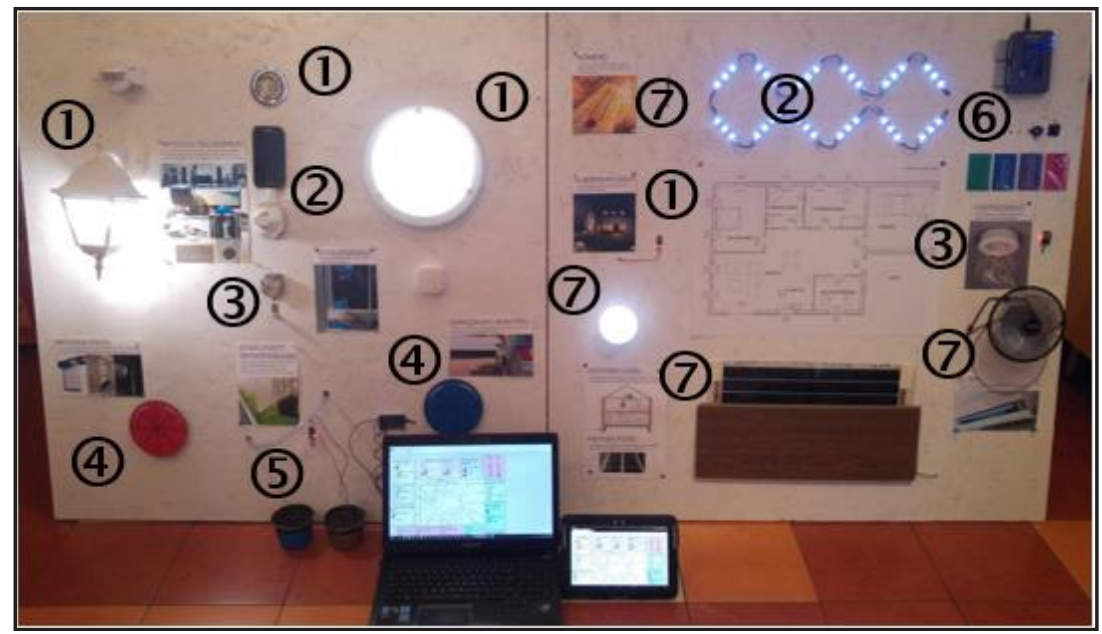

Fig. 8 - My home model that simulates real-life like conditions

\section{Conclusions}

I have created a model of a smart home that simulates reallife like conditions. This model meets the criteria that I have made about an "intelligent" house. There are automatic lighting, heating, cooling and sprinkling systems. There are also motorized controls and an advanced protection against robbery or fire.

The users can control the home with a user interface. This user interface is accessible through the web.

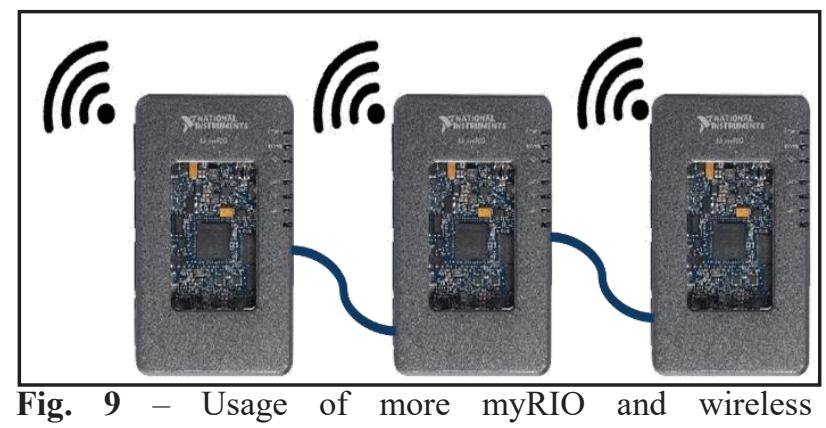

communication

In the future I would like to work on improvements and more functions. With my technique any standard need can be satisfied. However there can be extreme needs. myRIO has 40 digital input-output, 10 analogue input and 6 analogue output ports. If a user has such specific needs that require more ports than that, I would like to find the way using more myRIO-s in serial or parallel configuration.

Other solution would be using wireless sensors and actuators. In this way I need to learn in details about wireless protocols and how to use them effectively.

\section{References}

1. G. András, Industry 4.0 and Hungary, Ipar 4.0 és Magyarország, lecture record, Eger, (2017)

2. P. Kádár, The Smart home, A Smart lakás, Óbuda University, Budapest, (2012) ISBN: 978-615-501849-7

3. Prof. Dr. L. Monostori, I. Lepényesi, I. Ács, A, Oláh, M. Petrik, Prof C. Hassan, Industry 4.0 National Technological Platform, Ipar 4.0 Nemzeti Technológiai Platform, Abailable: https://www.i40platform.hu/

4. R. Haddad, How can our home be Smart Home?, Hogyan lehet otthonunk Smart Home?, Óbuda University, Budapest, (2012) ISBN: 978-615-501849-7 